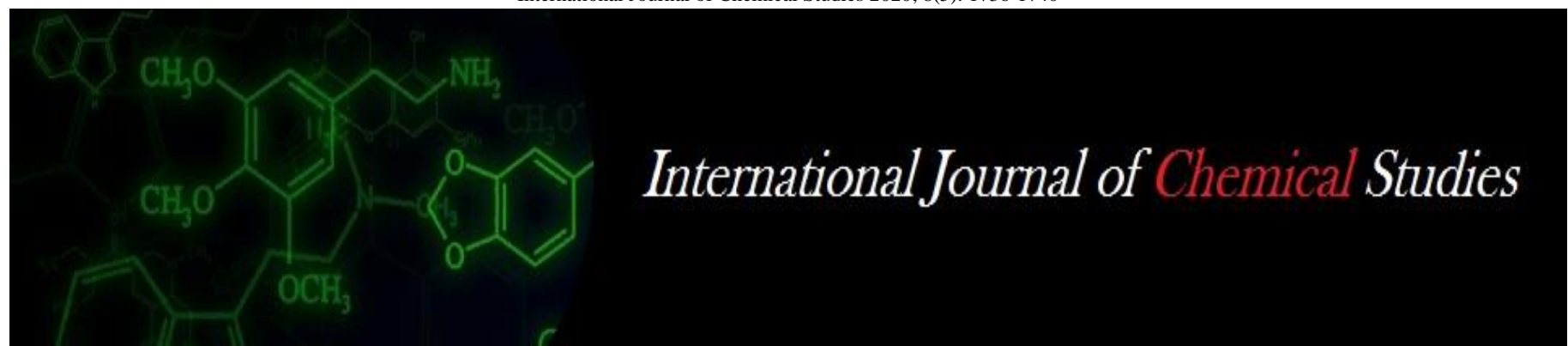

P-ISSN: 2349-8528

E-ISSN: 2321-4902

www.chemijournal.com

IJCS 2020; 8(5): 1736-1740

(C) 2020 IJCS

Received: 08-06-2020

Accepted: 15-07-2020

\section{AA Maheta}

Department of Agricultural Chemistry and Soil Science, Junagadh Agricultural

University, Junagadh, Gujarat, India

\section{JJ Vaghani}

Department of Agricultural Chemistry and Soil Science, Junagadh Agricultural

University, Junagadh, Gujarat, India

\section{AH Bavaliya}

Department of Agricultural Chemistry and Soil Science, Junagadh Agricultural University, Junagadh, Gujarat, India

\section{KC Gajera}

Department of Agricultural Chemistry and Soil Science, Junagadh Agricultural

University, Junagadh, Gujarat, India
Corresponding Author: AA Maheta

Department of Agricultural Chemistry and Soil Science, Junagadh Agricultural

University, Junagadh, Gujarat, India

\section{Effect of potassium (K) and potassium solubilizing bacteria (KSB) growth, yield, quality parameters and nutrient uptake by wheat (Triticum aestivum L.)}

\author{
AA Maheta, JJ Vaghani, AH Bavaliya and KC Gajera
}

DOI: https://doi.org/10.22271/chemi.2020.v8.i5x.10547

\begin{abstract}
A field experiment entitled Effect of potassium and potassium solubilizing bacteria (KSB) on growth, yield, quality parameters and nutrient content of wheat (Triticum aestivum L.) Var. GW 366 was conducted on clayey soils of Junagadh having status of low available nitrogen and medium available phosphorus and potassium during rabi season of 2017-18 at Instructional Farm, Department of Agronomy, College of Agriculture, Junagadh Agricultural University, Junagadh. The experiment comprised of 16 treatments having four levels of potassium $(\mathrm{K})\left(0,30,45\right.$ and $\left.60 \mathrm{~kg} \mathrm{~K}_{2} \mathrm{O} \mathrm{ha}{ }^{-1}\right)$ and four levels of potassium solubilizing bacteria $(\mathrm{KSB})\left(0,0.5,1.0\right.$ and $\left.1.5 \mathrm{lit}^{\mathrm{h}} \mathrm{a}^{-1}\right)$ carried out in factorial randomized block design (FRBD) replicated at thrice. The experimental results revealed that application of $60 \mathrm{~kg} \mathrm{~K}_{2} \mathrm{O} \mathrm{ha}^{-1}\left(\mathrm{~K}_{3}\right)$ with $\mathrm{KSB} 1$ lit ha ${ }^{-1}\left(\mathrm{KSB}_{2}\right)$ as soil application promoted growth parameters viz., grain \& straw yield, number of effective tillers per meter row, total tillers per meter row, spikelet's per spike, spike length and also quality parameter like test weight, protein content was found significant influenced by $60 \mathrm{~kg} \mathrm{~K}_{2} \mathrm{O} \mathrm{ha}^{-1}$ with KSB 1 lit ha $^{-1}\left(\mathrm{KSB}_{2}\right)$ recorded this treatment over the $0 \mathrm{~kg} \mathrm{~K}_{2} \mathrm{O} \mathrm{ha}^{-1}$. Potassium content in straw and grain at all stages significantly affected by $60 \mathrm{~kg} \mathrm{~K}_{2} \mathrm{O}^{-1}$ with $\mathrm{KSB} 1$ lit $\mathrm{ha}^{-1}\left(\mathrm{KSB}_{2}\right)$ over control.
\end{abstract}

Keywords: Wheat, potassium and KSB, growth, yield, nutrient content

\section{Introduction}

Wheat is one of the most important grain crops which is belongs to Poaceae family and staple food crop of the world and emerged as the backbone of India's food security. Wheat is one of the second most significant cereal in India following rice, contributing substantially to the national food security by providing more than $50 \%$ of the calories to the people who mainly depend on it. It is grown all over the world for its wider adaptability and high nutritive value. In Gujarat, wheat occupies an area of 1.06 million hectares with a production of 3.052 million tonnes and productivity of 2.89 metric tones $\mathrm{ha}^{-1}$ (Anon., 2018) ${ }^{[2]}$.

Potassium is one of 17 vital nutrients required for the growth and reproduction. Most of the $\mathrm{K}$ in soil exists in various insoluble rocks, minerals and sedimentary materials. Excessive usage of fertilizers leads to the leaching of nutrients from the soil and contributes to environmental pollution, without corresponding increases in yield. Potassium fixed with in soil and not easily meet to crop. Although most agricultural soils have large amounts of $\mathrm{K}$, these are immobilized and mostly become unavailable. Hence, very limited concentration of $\mathrm{K}$ is available to plants due to this $\mathrm{K}$ is deficient in soils. The amount of potassium present in the soil solution is often smaller than the wheat crop requirement for potassium. With the use of $\mathrm{K}$ solublizing microbes the concentration of available $\mathrm{K}$ ions can be increase in the soil which may further reduce $\mathrm{K}$ deficiency (Barker et al., 1998) ${ }^{[5]}$.

There is many research has done in previous year on KSB and their working mechanism. The use of KSB as a biological fertilizer is a hotspot for the study of agriculture and environmental conservation (Deng et al., 2003) ${ }^{[6]}$. The application of organo-minerals with the combination of silicate bacteria for enhancing plant growth and yield of maize and wheat first reported by Aleksandrov et al. (1967) ${ }^{[1]}$. Therefore, the use of KSB in agricultural practice would not only offset the high cost of manufacturing potassic fertilizers but would also mobilize insoluble 
fertilizers in the soils to which they are applied. It would also mobilize insoluble fertilizers in the soils to which they are applied. Use of phosphate bio-fertilizers decreases the detrimental effects of phosphate fertilizers on crop and soil health.

\section{Materials and Methods}

The field experiment was conducted during 2017-18 at Instructional Farm, Department of Agronomy, JAU, Junagadh situated at at $21.5^{\circ} \mathrm{N}$ latitude and $70.5^{\circ} \mathrm{E}$ longitude with an altitude of $60 \mathrm{~m}$ above the mean sea level on the wastern side at the foothill of mountain 'Girnar' under South Saurashtra Agro-climatic region of Gujarat. The soil of experimental site was clayey in texture with $\mathrm{pH} 8.1$ and EC $0.52 \mathrm{dS} \mathrm{m}^{-1}$. The soil was low in available nitrogen $\left(245.00 \mathrm{~kg} \mathrm{ha}^{-1}\right)$, medium in available phosphorus (41.10 $\left.\mathrm{kg} \mathrm{ha}^{-1}\right)$ and potassium (279.00 $\left.\mathrm{kg} \mathrm{ha}^{-1}\right)$. The experiment was laid out in factorial randomized block design with three replications. The experiment comprised of 16 treatments having four levels of potassium (K) $\left(0,30,45\right.$ and $\left.60 \mathrm{~kg} \mathrm{~K} \mathrm{~K}_{2} \mathrm{O} \mathrm{ha}^{-1}\right)$ and four levels of potassium soluiblizing bacteria $(\mathrm{KSB})(0,0.5,1.0$ and 1.5 lit $\mathrm{ha}^{-1}$ ) carried out in factorial randomized block design (FRBD) replicated at thrice and variety is GW 366.

\section{Results and Discussion}

(1) Yield and yield parameter (Grain \& Straw yield)

(A) Effect of potassium (K) \& KSB

A perusal of data revealed that different levels of potassium exhibited their significant influence on grain yield. The application of $60 \mathrm{~kg} \mathrm{~K}_{2} \mathrm{O} \mathrm{ha} \mathrm{ha}^{-1}\left(\mathrm{~K}_{3}\right)$ was recorded significantly the highest grain yield (4561 $\left.\mathrm{kg} \mathrm{ha}^{-1}\right) \&$ straw yield (6717) which was statistically equivalent to the treatment comprising soil application of $45 \mathrm{~kg} \mathrm{~K}_{2} \mathrm{O} \mathrm{ha}{ }^{-1}\left(\mathrm{~K}_{2}\right)$. While in $\mathrm{KSB}$, significantly the highest grain yield $\left(4475 \mathrm{~kg} \mathrm{ha}^{-1}\right)$ \& straw yield (5881) was recorded with application of KSB 1 lit ha ${ }^{-1}$ soil application $\left(\mathrm{KSB}_{2}\right)$, which was found statistically at par with application of KSB 1.5 lit ha ${ }^{-1}$ soil inoculation $\left(\mathrm{KSB}_{3}\right)$.

This results also close conformity with Archna et al. (2007) reported that $\mathrm{KSB}$ is able to solubilize inorganic source of $\mathrm{K}$ like muriate of potash by means of production of organic acids in order to improve yield in maize crop. Bagyalakshami et al. (2012) ${ }^{[4]}$ reported that application of indigenous KSB formulation with various doses of potash fertilizers with $\mathrm{N}$ and $\mathrm{P}$ enhanced green leaf yield and productivity in tea. The increase in yield might be due to the solubilization of nutrients in the soil by producing organic acids by KSB.

Possible direct effect of the bacteria on plant growth via phytohormones, the bacteria mainly acted by an indirect way via mineral weathering, which increased the amount of available nutrients for the plant and/or a direct effect of the bacteria on plant roots by phytohormones which stimulate the formation of lateral roots and absorbent root hairs (Mikhailouskaya et al. (2009) ${ }^{[8]}$ in winter rye, spring wheat and winter triticale, Zhanga et al. (2013) [11] in okra and Konga (2014) ${ }^{[7]}$ in tobacco.

Table 1: Effect of potassium and KSB levels on yield, growth and quality parameters of wheat

\begin{tabular}{|c|c|c|c|c|c|c|c|c|}
\hline Treatments & \begin{tabular}{|c|}
$\begin{array}{c}\text { Grain yield } \\
\left(\mathrm{kg} \mathrm{ha}^{-1}\right)\end{array}$ \\
\end{tabular} & \begin{tabular}{|c} 
Straw yield \\
$\left(\mathrm{kg} \mathrm{ha}^{-1}\right)$
\end{tabular} & \begin{tabular}{|c|}
$\begin{array}{c}\text { No. of effective tillers } \\
\text { per meter row }\end{array}$ \\
\end{tabular} & \begin{tabular}{|c|}
$\begin{array}{c}\text { No. of total tillers } \\
\text { per meter row }\end{array}$ \\
\end{tabular} & \begin{tabular}{|c|} 
Spikelet \\
per spike
\end{tabular} & \begin{tabular}{|c|}
$\begin{array}{c}\text { Spike length } \\
(\mathrm{cm})\end{array}$ \\
\end{tabular} & \begin{tabular}{|c|} 
Test \\
weight $(\mathrm{g})$ \\
\end{tabular} & $\begin{array}{c}\text { Protein } \\
\text { content }(\%)\end{array}$ \\
\hline \multicolumn{9}{|c|}{ Potassium (K) } \\
\hline $\mathrm{K}_{0}: 0 \mathrm{~kg} \mathrm{~K}_{2} \mathrm{O} \mathrm{ha}^{-1}$ & 3600 & 4246 & 52.43 & 73.85 & 12.82 & 6.78 & 50.29 & 10.41 \\
\hline $\mathrm{K}_{1}: 30 \mathrm{~kg} \mathrm{~K}_{2} \mathrm{O} \mathrm{ha}^{-1}$ & 3660 & 5137 & 54.26 & 80.33 & 13.98 & 7.22 & 52.45 & 10.80 \\
\hline $\mathrm{K}_{2}: 45 \mathrm{~kg} \mathrm{~K}_{2} \mathrm{O} \mathrm{ha}^{-1}$ & 4458 & 6374 & 72.63 & 86.43 & 15.28 & 8.23 & 59.48 & 11.52 \\
\hline $\mathrm{K}_{3}: 60 \mathrm{~kg} \mathrm{~K}_{2} \mathrm{O} \mathrm{ha}^{-1}$ & 4561 & 6717 & 75.99 & 88.83 & 16.06 & 8.62 & 61.03 & 11.94 \\
\hline S.Em \pm & 107 & 140 & 1.22 & 2.17 & 0.35 & 0.25 & 0.84 & 0.41 \\
\hline C.D. at $5 \%$ & 310 & 403 & 3.53 & 6.28 & 1.03 & 0.73 & 2.42 & NS \\
\hline \multicolumn{9}{|c|}{ Potassium solublizing bacteria (KSB) } \\
\hline $\mathrm{KSB}_{0}:$ KSB 0 lit ha' & 3614 & 5339 & 58.60 & 76.90 & 14.10 & 7.43 & 54.02 & 10.61 \\
\hline $\mathrm{KSB}_{1}:$ KSB 0.5 lit ha $^{-1}$ & 3864 & 5441 & 61.38 & 80.27 & 14.34 & 7.49 & 54.28 & 10.69 \\
\hline $\mathrm{KSB}_{2}:$ KSB 1 lit ha ${ }^{-1}$ & 4475 & 5881 & 71.66 & 88.11 & 14.87 & 8.17 & 57.99 & 12.05 \\
\hline $\mathrm{KSB}_{3}:$ KSB 1.5 lit ha $\mathrm{h}^{-1}$ & 4326 & 5812 & 68.68 & 84.22 & 14.83 & 7.77 & 56.96 & 11.32 \\
\hline S.Em \pm & 107 & 140 & 1.22 & 2.17 & 0.35 & 0.25 & 0.84 & 0.41 \\
\hline C.D. at $5 \%$ & 310 & 403 & 3.53 & 6.28 & NS & NS & 2.42 & NS \\
\hline \multicolumn{9}{|c|}{ K x KSB } \\
\hline S.Em \pm & 261 & 279 & 2.44 & 4.35 & 0.71 & 0.51 & 1.68 & 0.81 \\
\hline C.D. at $5 \%$ & 755 & NS & 7.06 & NS & NS & NS & NS & NS \\
\hline $\mathrm{CV} \%$ & 11.90 & 8.61 & 6.50 & 9.14 & 8.46 & 11.40 & 5.20 & 12.64 \\
\hline
\end{tabular}

\section{(B) Interaction effect of potassium (K) and KSB}

Examination of data showed that interaction effect of different levels of potassium and KSB on grain yield was found significant. Wherein, the treatment combination $\mathrm{K}_{3} \mathrm{KSB}_{2}(60$ $\mathrm{kg} \mathrm{K} \mathrm{K}_{2} \mathrm{O} \mathrm{ha}^{-1}+\mathrm{KSB} 1$ lit $\mathrm{ha}^{-1}$ ) recorded significantly the highest grain yield $\left(5184 \mathrm{~kg} \mathrm{ha}^{-1}\right)$, however it remained statistically at par with the treatment combinations $\mathrm{K}_{2} \mathrm{KSB}_{3}$ $\left(45 \mathrm{~kg} \mathrm{~K}_{2} \mathrm{O} \mathrm{ha}^{-1}+\mathrm{KSB} 1.5 \mathrm{lit} \mathrm{ha}^{-1}\right), \mathrm{K}_{2} \mathrm{KSB}_{2}\left(45 \mathrm{~kg} \mathrm{~K}_{2} \mathrm{O} \mathrm{ha}^{-1}\right.$ $\left.+\mathrm{KSB} 1 \mathrm{lit} \mathrm{ha}^{-1}\right)$ and $\mathrm{K}_{3} \mathrm{KSB}_{3}\left(60 \mathrm{~kg} \mathrm{~K}_{2} \mathrm{O} \mathrm{ha}^{-1}+\mathrm{KSB} 1.5\right.$ lit $\left.\mathrm{ha}^{-1}\right)$. Whereas, the treatment combination $\mathrm{K}_{0} \mathrm{KSB}_{0}\left(0 \mathrm{~kg} \mathrm{~K} \mathrm{~K}_{2} \mathrm{O}\right.$ $\mathrm{ha}^{-1}+\mathrm{KSB} 0$ lit $\mathrm{ha}^{-1}$ ) registered significantly lowest grain yield. While in straw yield interaction found non significant.
Table 2: Interaction effect of potassium and KSB levels on grain yield $\left(\mathrm{kg} \mathrm{ha}^{-1}\right)$ of wheat

\begin{tabular}{|c|c|c|c|c|}
\hline KSB (lit ha-1) & & & & \\
\hline $\begin{array}{c}\text { Potassium }(k g \\
\left.\text { ha }^{-1}\right)\end{array}$ & $\begin{array}{c}\text { KSBO } \\
\text { (KSB 0) }\end{array}$ & $\begin{array}{c}\text { KSB1(KSB } \\
0.5)\end{array}$ & $\begin{array}{c}\text { KSB }_{2} \text { (KSB } \\
1.0)\end{array}$ & $\begin{array}{c}\text { KSB3(KSB } \\
1.5)\end{array}$ \\
\hline $\mathrm{K}_{0}\left(0 \mathrm{~K}_{2} \mathrm{O}\right)$ & 3382 & 3617 & 3793 & 3608 \\
\hline $\mathrm{K}_{1}\left(30 \mathrm{~K}_{2} \mathrm{O}\right)$ & 3395 & 3772 & 3826 & 3647 \\
\hline $\mathrm{K}_{2}\left(45 \mathrm{~K}_{2} \mathrm{O}\right)$ & 3797 & 3926 & 5097 & 5014 \\
\hline $\mathrm{K}_{3}\left(60 \mathrm{~K}_{2} \mathrm{O}\right)$ & 3883 & 4141 & 5184 & 5034 \\
\hline S.Em \pm & \multicolumn{4}{|c|}{214} \\
\hline C.D. at $5 \%$ & \multicolumn{4}{|c|}{619} \\
\hline
\end{tabular}




\section{(2) Growth parameter}

\section{(A) Effect of potassium (K) \& KSB}

Significantly the highest number of effective tillers $\mathrm{m}^{-1}$ (75.99) spikelet per spike (16.06), total tillers $\mathrm{m}^{-1}$ (88.83), spike length $(8.62 \mathrm{~cm})$ was showed with application of $60 \mathrm{~kg}$ $\mathrm{K}_{2} \mathrm{O}$ ha $^{-1}\left(\mathrm{~K}_{3}\right)$, which was statistically equivalent to the treatment comprising soil application of $45 \mathrm{~kg} \mathrm{~K}_{2} \mathrm{O} \mathrm{ha}^{-1}\left(\mathrm{~K}_{2}\right)$. On the contrary, the $0 \mathrm{~kg} \quad \mathrm{~K}_{2} \mathrm{O} \quad \mathrm{ha}^{-1} \quad\left(\mathrm{~K}_{0}\right)$ registered significantly the lowest result. Patel et al. (2007) ${ }^{[9]}$ showed that the application of potassium $60 \mathrm{~kg} \mathrm{~K}_{2} \mathrm{O} \mathrm{ha}{ }^{-1}$ significantly increased the number of effective tillers per meter row length (81.41) over control and $30 \mathrm{~kg} \mathrm{~K}_{2} \mathrm{O} \mathrm{ha}^{-1}$. Patel et al. (2007) ${ }^{[9]}$ showed that the application of potassium $60 \mathrm{~kg} \mathrm{~K} \mathrm{~K}_{2} \mathrm{O} \mathrm{ha}^{-1}$ significantly increased the number of effective tillers per meter row length (81.41) over control and $30 \mathrm{~kg} \mathrm{~K}_{2} \mathrm{O} \mathrm{ha}^{-1}$.

With regard to observation treatment $\mathrm{KSB} 1$ lit ha ${ }^{-1}$ soil application $\left(\mathrm{KSB}_{2}\right)$ established its superiority by recording significantly the highest number of effective tillers $\mathrm{m}^{-1}$ row (71.66), total tillers $\mathrm{m}^{-1}$ (88.11) \& Various levels of KSB did not compel significant influence on number of spikelet per spike \& spike length.

\section{(B) Interaction effect of potassium (K) \& KSB}

Combined effect of potassium \& KSB found non significant on growth parameter except no. of effective tillers per meter row.

\section{(3) Quality parameter}

\section{(A) Effect of potassium (K) \& KSB}

A perusal of data revealed that different levels of potassium indicated their significant influence on test weight. Significantly the highest 1000 -grain weight $(61.03 \mathrm{~g})$ was noticed with treatment of $60 \mathrm{~kg} \mathrm{~K}_{2} \mathrm{O} \mathrm{ha}^{-1}\left(\mathrm{~K}_{3}\right)$, which was statistically at par to the treatment comprising soil application of $45 \mathrm{~kg} \mathrm{~K}_{2} \mathrm{O}$ ha $^{-1}\left(\mathrm{~K}_{2}\right)$.

Significantly the highest test weight (57.99 g) was exerted with treatment of KSB 1 lit ha ${ }^{-1}$ soil application $\left(\mathrm{KSB}_{2}\right)$, which was found statistically comparable with application of KSB 1.5 lit ha ${ }^{-1}$ soil inoculation $\left(\mathrm{KSB}_{3}\right)$.
Scutiny of data showed that interaction effect of different levels of potassium and KSB was found non-significant on test weight.

An examination of data revealed that different levels of potassium, KSB \& their did not exert their significant influence on protein content.

Table 3: Interaction effect of potassium and KSB levels on no. of effective tillers $\mathrm{m}^{-1}$ row of wheat

\begin{tabular}{|c|c|c|c|c|}
\hline KSB (lit ha-1) & $\overline{\mathrm{KSB}_{0}}$ & $\overline{\text { KSB }_{1}}$ & $\overline{\mathrm{KSB}_{2}}$ & $\mathrm{KSB}_{3}$ \\
\hline $\operatorname{Potassium}\left(\mathrm{kg} \mathrm{ha}^{-1}\right)$ & (KSB 0) & (KSB 0.5) & (KSB 1.0) & (KSB 1.5) \\
\hline $\mathrm{K}_{0}\left(0 \mathrm{~K}_{2} \mathrm{O}\right)$ & 42.47 & 51.73 & 58.63 & 56.90 \\
\hline $\mathrm{K}_{1}\left(30 \mathrm{~K}_{2} \mathrm{O}\right)$ & 56.93 & 57.30 & 61.53 & 61.27 \\
\hline $\mathrm{K}_{2}\left(45 \mathrm{~K}_{2} \mathrm{O}\right)$ & 63.27 & 63.87 & 82.93 & 80.47 \\
\hline $\mathrm{K}_{3}\left(60 \mathrm{~K}_{2} \mathrm{O}\right)$ & 71.73 & 72.63 & 83.53 & 76.07 \\
\hline S.Em \pm & \multicolumn{4}{|c|}{2.44} \\
\hline C.D. at $5 \%$ & \multicolumn{4}{|c|}{7.06} \\
\hline
\end{tabular}

\section{(4) Nutrient content in wheat}

\section{(A) Effect of potassium \& KSB}

As evident of data show that not significantly effect on the most nitrogen and phosphorus content in grain \& straw found non significant at different levels of potassium and KSB. Various treatments of potassium did exhibit their significant influence on potassium content in straw at 30, 60, 90 DAS and at harvest (grain \& straw). Among different treatments of potassium, application of $60 \mathrm{~kg} \mathrm{~K} \mathrm{~K}_{2} \mathrm{O} \mathrm{ha}^{-1}\left(\mathrm{~K}_{3}\right)$ recorded significantly the highest potassium content in straw and grain. While Potassium content in straw at 30 DAS remained unaffected under KSB levels. While, under the given treatment schedule treatment KSB 1 lit ha ${ }^{-1}\left(\mathrm{KSB}_{2}\right)$ potassium content in straw 60, 90 DAS and at harvest (grain \& straw) recorded significant.

\section{(B) Interaction effect of potassium \& $(\mathrm{KSB})$}

Combined effect between different levels of potassium and KSB failed to manifest significant effect on nitrogen, phosphorus and potassium content in straw and grain.

Table 4: Effect of potassium and KSB levels on nutrient content (Nitrogen, Phosphorus, Potassium) of wheat

\begin{tabular}{|c|c|c|c|c|c|c|c|c|c|c|c|c|}
\hline \multirow{3}{*}{ Treatments } & \multicolumn{7}{|c|}{ Potassium content (\%) } & \multirow{2}{*}{\multicolumn{3}{|c|}{$\begin{array}{c}\text { Phosphorus content (\%) } \\
\text { At harvest }\end{array}$}} & \multirow{2}{*}{\multicolumn{2}{|c|}{$\begin{array}{c}\text { Nitrogen content }(\%) \\
\text { At harvest }\end{array}$}} \\
\hline & \multirow{2}{*}{\multicolumn{2}{|c|}{30 DAS }} & \multirow{2}{*}{\multicolumn{2}{|c|}{60 DAS }} & \multirow{2}{*}{90 DAS } & \multicolumn{2}{|c|}{ At harvest } & & & & & \\
\hline & & & & & & Grain & Straw & \multicolumn{2}{|c|}{\begin{tabular}{c|c}
\multicolumn{2}{c}{ At harvest } \\
Grain & Straw \\
\end{tabular}} & \multicolumn{2}{|c|}{ Grain } & \multirow[t]{2}{*}{ Straw } \\
\hline \multicolumn{12}{|c|}{ Potassium (K) } & \\
\hline \multicolumn{2}{|c|}{$\mathrm{K}_{0}: 0 \mathrm{~kg} \mathrm{~K}_{2} \mathrm{O} \mathrm{ha}^{-1}$} & & .77 & 2.15 & 1.69 & 0.383 & 1.17 & 0.358 & & 0.120 & 1.67 & 0.368 \\
\hline \multicolumn{2}{|c|}{$\mathrm{K}_{1}: 30 \mathrm{~kg} \mathrm{~K}_{2} \mathrm{O} \mathrm{ha}^{-1}$} & & .82 & 2.21 & 1.72 & 0.420 & 1.23 & 0.359 & & 0.131 & 1.73 & 0.401 \\
\hline \multicolumn{2}{|c|}{$\mathrm{K}_{2}: 45 \mathrm{~kg} \mathrm{~K}_{2} \mathrm{O} \mathrm{ha}^{-1}$} & & .96 & 2.38 & 1.87 & 0.518 & 1.31 & 0.399 & & 0.139 & 1.84 & 0.411 \\
\hline \multicolumn{2}{|c|}{$\mathrm{K}_{3}: 60 \mathrm{~kg} \mathrm{~K}_{2} \mathrm{O} \mathrm{ha}^{-1}$} & & .04 & 2.42 & 1.89 & 0.524 & 1.35 & 0.400 & & 0.140 & 1.91 & 0.418 \\
\hline \multicolumn{2}{|l|}{ S.Em \pm} & & .04 & 0.04 & 0.03 & 0.013 & 0.02 & 0.015 & & 0.006 & 0.07 & 0.017 \\
\hline \multicolumn{2}{|c|}{ C.D. at $5 \%$} & & .13 & 0.11 & 0.08 & 0.037 & 0.06 & NS & & NS & NS & NS \\
\hline \multicolumn{13}{|c|}{ Potassium solublizing bacteria (KSB) } \\
\hline \multicolumn{2}{|c|}{$\mathrm{KSB}_{0}:$ KSB 0 lit ha ${ }^{-1}$} & 2.8 & & 2.21 & 1.72 & 0.421 & 1.22 & 0.346 & & 0.122 & 1.70 & 0.367 \\
\hline $\mathrm{KSB}_{1}: \mathrm{KSB} 0.5$ & & 2.9 & & 2.24 & 1.76 & 0.440 & 1.24 & 0.378 & & 0.132 & 1.71 & 0.393 \\
\hline $\mathrm{KSB}_{2}: \mathrm{KSB} 1 \mathrm{li}$ & & 2.9 & & 2.37 & 1.87 & 0.501 & 1.32 & 0.402 & & 0.143 & 1.93 & 0.423 \\
\hline $\mathrm{KSB}_{3}: \mathrm{KSB} 1.5$ & $\mathrm{a}^{-1}$ & 2.9 & & 2.34 & 1.82 & 0.484 & 1.28 & 0.391 & & 0.132 & 1.81 & 0.415 \\
\hline S.Em \pm & & 0.0 & & 0.04 & 0.03 & 0.013 & 0.02 & 0.015 & & 0.006 & 0.07 & 0.017 \\
\hline C.D. at $5 \%$ & & $\mathrm{Ns}$ & & 0.11 & 0.08 & 0.037 & 0.06 & NS & & NS & NS & NS \\
\hline & & & & & & & $\mathrm{x} \mathrm{KSB}$ & & & & & \\
\hline S.Em \pm & & 0.09 & & 0.08 & \begin{tabular}{|l|l|}
8 & 0.05 \\
\end{tabular} & 0.025 & 0.04 & 0.029 & & 0.012 & \begin{tabular}{|l|}
0.13 \\
\end{tabular} & 0.034 \\
\hline C.D. at 5\% & & $\mathrm{NS}$ & & $\mathrm{NS}$ & \begin{tabular}{|l|l|} 
& NS \\
\end{tabular} & NS & NS & NS & & $\mathrm{NS}$ & NS & $\mathrm{NS}$ \\
\hline $\mathrm{CV} \%$ & & 5.28 & & 5.71 & \begin{tabular}{|l|l|}
1 & 5.25 \\
\end{tabular} & 9.55 & 5.86 & 13.42 & & 15.61 & 12.64 & 14.69 \\
\hline
\end{tabular}


(5) Nutrient uptake by wheat

(A) Effect of potassium \& KSB

Different fertilization of potassium and KSB treatments produce significant effect on nitrogen, phosphorus and potassium $(30,60,90$ and at harvest) uptake by straw \& grain. Significantly the highest nitrogen, phosphorus and potassium (30, 60, 90 and at harvest) uptake by grain and straw was recorded with the treatment $\mathrm{K}_{3}\left(60 \mathrm{~kg} \mathrm{~K}_{2} \mathrm{O}\right.$ ha $\left.^{-1}\right)$ with $\mathrm{KSB} 1$ lit ha ${ }^{-1}\left(\mathrm{KSB}_{2}\right)$, but it was found statistically at par with the treatments $\mathrm{K}_{2}\left(45 \mathrm{~kg} \mathrm{~K}_{2} \mathrm{O} \mathrm{ha}^{-1}\right)$ with $\mathrm{KSB} 1.5$ lit ha $^{-1}$ soil inoculation $\left(\mathrm{KSB}_{3}\right)$.

Sheng et al. (2003) ${ }^{[10]}$ reported that in cotton and rapeseed root and shoot growth and potassium content was increased by 30 and 26 percent respectively and in chilli crop increased biomass and $\mathrm{K}$ uptake due to inoculation of potash solubilizer in China.

\section{(B) Interaction effect of potassium \& (KSB)}

The interaction effect of different levels of potassium and KSB was found non-significant as far as nitrogen, phosphorus and potassium uptake by straw \& grain is concerned. Except nitrogen uptake by grain and potassium uptake by straw at 90 DAS found significant at different levels potassium and KSB levels.

Table 5: Effect of potassium and KSB levels on nutrient uptake (Nitrogen, Phosphorus, Potassium) by wheat

\begin{tabular}{|c|c|c|c|c|c|c|c|c|c|}
\hline \multirow{3}{*}{ Treatments } & \multicolumn{5}{|c|}{ Potassium uptake $\left(\right.$ mg plant $\left.^{-1}\right)$} & \multirow{2}{*}{\multicolumn{2}{|c|}{$\begin{array}{l}\text { Phosphorus uptake } \\
\text { At harvest }\left(\mathrm{kg} \mathrm{ha}^{-1}\right)\end{array}$}} & \multirow{2}{*}{\multicolumn{2}{|c|}{$\begin{array}{c}\text { Nitrogen uptake } \\
\text { At harvest }\left(\mathrm{kg} \mathrm{ha}^{-1}\right)\end{array}$}} \\
\hline & \multirow{2}{*}{30 DAS } & \multirow{2}{*}{60 DAS } & \multirow{2}{*}{90 DAS } & \multicolumn{2}{|c|}{ At harvest $\left(\mathrm{kg} \mathrm{ha}^{-1}\right)$} & & & & \\
\hline & & & & Grain & Straw & Grain & Straw & Grain & Straw \\
\hline \multicolumn{10}{|c|}{ Potassium $(\mathbf{K})$} \\
\hline $\mathrm{K}_{0}: 0 \mathrm{~kg} \mathrm{~K}_{2} \mathrm{O} \mathrm{ha}^{-1}$ & 43.61 & 162.5 & 239.00 & 13.81 & 49.33 & 12.94 & 5.10 & 60.04 & 15.62 \\
\hline $\mathrm{K}_{1}: 30 \mathrm{~kg} \mathrm{~K}_{2} \mathrm{O} \mathrm{ha}^{-1}$ & 61.82 & 188.7 & 276.99 & 15.38 & 63.20 & 13.13 & 6.78 & 63.31 & 20.72 \\
\hline $\mathrm{K}_{2}: 45 \mathrm{~kg} \mathrm{~K}_{2} \mathrm{O} \mathrm{ha}^{-1}$ & 93.71 & 228.2 & 355.51 & 23.34 & 83.30 & 17.91 & 8.83 & 83.55 & 26.07 \\
\hline $\mathrm{K}_{3}: 60 \mathrm{~kg} \mathrm{~K}_{2} \mathrm{O} \mathrm{ha}^{-1}$ & 99.66 & 240.4 & 368.75 & 24.15 & 90.72 & 18.51 & 9.40 & 87.50 & 28.05 \\
\hline S.Em \pm & 2.21 & 5.60 & 6.43 & 0.77 & 2.73 & 0.68 & 0.39 & 3.63 & 1.07 \\
\hline C.D. at $5 \%$ & 6.37 & 16.17 & 18.58 & 2.22 & 7.89 & 1.97 & 1.13 & 10.50 & 3.08 \\
\hline \multicolumn{10}{|c|}{ Potassium solublizing bacteria (KSB) } \\
\hline $\mathrm{KSB}_{0}$ : KSB 0 lit ha ${ }^{-1}$ & 64.16 & 184.18 & 286.57 & 15.33 & 65.36 & 12.83 & 6.62 & 61.58 & 19.52 \\
\hline $\mathrm{KSB}_{1}$ : KSB 0.5 lit ha $^{-1}$ & 72.80 & 195.87 & 294.33 & 17.14 & 68.09 & 15.03 & 7.19 & 66.08 & 21.55 \\
\hline $\mathrm{KSB}_{2}:$ KSB 1 lit ha ${ }^{-1}$ & 83.72 & 223.10 & 333.86 & 22.93 & 78.43 & 17.93 & 8.61 & 87.62 & 25.07 \\
\hline $\mathrm{KSB}_{3}: \mathrm{KSB} 1.5$ lit ha $^{-1}$ & 78.11 & 216.89 & 325.48 & 21.28 & 74.67 & 16.71 & 7.70 & 79.11 & 24.33 \\
\hline S.Em \pm & 2.21 & 5.60 & 6.43 & 0.77 & 2.73 & 0.68 & 0.39 & 3.63 & 1.07 \\
\hline C.D. at $5 \%$ & 6.37 & 16.17 & 18.58 & 2.22 & 7.89 & 1.97 & 1.13 & 10.50 & 3.08 \\
\hline \multicolumn{10}{|c|}{ K x KSB } \\
\hline S.Em \pm & 4.41 & 11.20 & 1.54 & 5.46 & 0.04 & 1.37 & 1.37 & 7.27 & 2.14 \\
\hline C.D. at $5 \%$ & NS & NS & 4.45 & NS & NS & NS & NS & 20.99 & NS \\
\hline CV\% & 10.23 & 9.46 & 13.92 & 13.21 & 5.86 & 15.15 & 15.15 & 17.10 & 16.35 \\
\hline
\end{tabular}

(6) Interaction effect of potassium and KSB on potassium uptake by wheat straw at 90 DAS

Examination of data notice that interaction effect of different levels of potassium and KSB on potassium uptake by grain was found significant. Wherein, the treatment combination $\mathrm{K}_{2} \mathrm{KSB}_{2}\left(45 \mathrm{~kg} \mathrm{~K}_{2} \mathrm{O} \mathrm{ha}^{-1}+\mathrm{KSB} 1\right.$ lit $\left.\mathrm{ha}^{-1}\right)$ recorded significantly the highest potassium uptake by grain $(30.67 \mathrm{~kg}$ $\left.\mathrm{ha}^{-1}\right)$, however it remained statistically at par with the treatment combinations $\mathrm{K}_{3} \mathrm{KSB}_{3}\left(60 \mathrm{~kg} \mathrm{~K}_{2} \mathrm{O}\right.$ ha $^{-1}+\mathrm{KSB} 1.5$ lit $\left.\mathrm{ha}^{-1}\right)$ and $\mathrm{K}_{3} \mathrm{KSB}_{3}\left(60 \mathrm{~kg} \mathrm{~K}_{2} \mathrm{O} \mathrm{ha}^{-1}+\mathrm{KSB} 1\right.$ lit ha $\left.{ }^{-1}\right)$. Whereas, the treatment combination $\mathrm{K}_{0} \mathrm{KSB}_{0}\left(0 \mathrm{~kg} \mathrm{~K}_{2} \mathrm{O} \mathrm{ha}^{-1}+\mathrm{KSB} 0\right.$ lit $\left.\mathrm{ha}^{-1}\right)$ registered significantly the lowest potassium uptake by grain $\left(12.00 \mathrm{~kg} \mathrm{ha}^{-1}\right)$.

Table 6: Potassium uptake by grain

\begin{tabular}{|c|c|c|c|c|}
\hline KSB (lit ha $\left.{ }^{-1}\right)$ & $\mathbf{K S B}_{0}(\mathrm{KSB} \mathbf{0})$ & $\mathrm{KSB}_{3}(\mathrm{KSB} 05)$ & $\mathrm{KSB}_{3}(\mathrm{KSB} 10)$ & $\mathrm{KSB}_{3}\left(\mathrm{KSB}_{1} \mathbf{5}^{2}\right.$ \\
\hline Potassium $\left(\mathrm{kg} \mathrm{ha}^{-1}\right)$ & KSBO & KSB1 (KSB 0.5) & KSB 2 (KSB 1.0) & KSB 3 (KSB 1.5) \\
\hline $\mathrm{K}_{0}\left(0 \mathrm{~K}_{2} \mathrm{O}\right)$ & 12.00 & 13.72 & 14.87 & 14.64 \\
\hline $\mathrm{K}_{1}\left(30 \mathrm{~K}_{2} \mathrm{O}\right)$ & 13.23 & 14.35 & 17.13 & 16.82 \\
\hline $\mathrm{K}_{2}\left(45 \mathrm{~K}_{2} \mathrm{O}\right)$ & 18.36 & 19.25 & 30.67 & 25.06 \\
\hline $\mathrm{K}_{3}\left(60 \mathrm{~K}_{2} \mathrm{O}\right)$ & 17.74 & 21.21 & 29.06 & 28.59 \\
\hline S.Em \pm & \multicolumn{4}{|c|}{1.54} \\
\hline C.D. at $5 \%$ & \multicolumn{4}{|c|}{4.45} \\
\hline
\end{tabular}

(7) Interaction effect of potassium and KSB on nitrogen uptake by wheat grain

An obvious from data presented that interactive effect of different levels of potassium and KSB was found significant as far as nitrogen uptake by grain is concerned. Wherein, the treatment combination $\mathrm{K}_{2} \mathrm{KSB}_{2}\left(45 \mathrm{~kg} \mathrm{~K}_{2} \mathrm{O}\right.$ ha $^{-1}+\mathrm{KSB} 1$ lit $\left.\mathrm{ha}^{-1}\right)$ recorded significantly the highest nitrogen uptake
(111.96 $\left.\mathrm{kg} \mathrm{ha}^{-1}\right)$ by grain, however it remained statistically at par with the treatment combinations $\mathrm{K}_{3} \mathrm{KSB}_{3}\left(60 \mathrm{~kg} \mathrm{~K} \mathrm{~K}_{2} \mathrm{Oa}^{-}\right.$ ${ }^{1}+\mathrm{KSB} 1.5$ lit ha $\left.^{-1}\right), \mathrm{K}_{3} \mathrm{KSB}_{2}\left(60 \mathrm{~kg} \mathrm{~K}_{2} \mathrm{O} \mathrm{ha} \mathrm{ha}^{-1}+\mathrm{KSB} 1\right.$ lit ha $\left.{ }^{-1}\right)$ and $\mathrm{K}_{2} \mathrm{KSB}_{3}\left(45 \mathrm{~kg} \mathrm{~K}_{2} \mathrm{O} \mathrm{ha}^{-1}+\mathrm{KSB} 1.5\right.$ lit ha $\left.{ }^{-1}\right)$. Whereas, the treatment combination $\mathrm{K}_{0} \mathrm{KSB}_{0}\left(0 \mathrm{~kg} \mathrm{~K}_{2} \mathrm{O} \mathrm{ha}^{-1}+\mathrm{KSB}_{0}\right.$ lit ha$\left.{ }^{1}\right)$ registered significantly least nitrogen uptake $\left(55.53 \mathrm{~kg} \mathrm{ha}^{-1}\right)$ by grain. 
Table 7: The treatment combination K0KSB0

\begin{tabular}{|c|c|c|c|c|}
\hline KSB $\left(\right.$ lit ha' $\left.{ }^{-1}\right)$ & & & & \\
\hline Potassium $\left(\mathrm{kg} \mathrm{ha}^{-1}\right)$ & $\mathrm{KSB}_{0}(\mathrm{KSB} \mathbf{0})$ & $\mathrm{KSB}_{1}$ (KSB 0.5) & $\mathrm{KSB}_{2}$ (KSB 1.0) & KSB $_{3}$ (KSB 1.5) \\
\hline $\mathrm{K}_{0}\left(0 \mathrm{~K}_{2} \mathrm{O}\right)$ & 55.53 & 60.32 & 63.31 & 60.99 \\
\hline $\mathrm{K}_{1}\left(30 \mathrm{~K}_{2} \mathrm{O}\right)$ & 57.82 & 64.59 & 67.98 & 62.85 \\
\hline $\mathrm{K}_{2}\left(45 \mathrm{~K}_{2} \mathrm{O}\right)$ & 58.32 & 66.28 & 111.96 & 97.64 \\
\hline $\mathrm{K}_{3}\left(60 \mathrm{~K}_{2} \mathrm{O}\right)$ & 74.67 & 73.12 & 107.24 & 94.94 \\
\hline S.Em \pm & \multicolumn{4}{|c|}{7.19} \\
\hline C.D. at $5 \%$ & \multicolumn{4}{|c|}{20.99} \\
\hline
\end{tabular}

\section{Conclusion}

It could be concluded that soil application of potassium at 60 $\mathrm{kg} \mathrm{K}_{2} \mathrm{O}$ ha $^{-1}$ and KSB 1 lit $\mathrm{ha}^{-1}$ enhanced grain yield and quality of wheat and maintaining soil fertility with saving of fertilizer dose of potash.

\section{References}

1. Aleksandrov VG, Blagodyr RN, Iiiev IP. Liberation of phosphoric acid from apatite by silicate bacteria. MikrobiyolZh. (Kiev). 1967; 29(3):111-114.

2. Anonymous. Pocket Book on Agricultural Statistics 2018. Ministry of Agriculture \& Farmers Welfare, Department of Agriculture and Cooperation \& Farmers Welfare, Government of India, New Delhi. Available at http://eands.dacnet.nic.in. accessed on, 2018.

3. Archana DS, Savalgi VP, Alagawadi AR. Effect of potassium soluiblizing bacteria on growth and yield of maize. Soil Biology and Ecology, 28(1-2): 9-18. Bacteria and KSB effects on plant growth. World Journal of Agriculture Science. 2008; 3(3):350-355.

4. Bagyalakshami $\mathrm{B}$, Ponmurugan $\mathrm{P}$, Marimuthu $\mathrm{S}$. Influence of potassium solubilizing bacteria on crop productivity and quality of tea (Camellia sinensis). African Journal of Agricultural Research. 2012; 7(30):4250-4259.

5. Barker WW, Welch SA, Chu S, Banfield F. Experimental observations of the effects of bacteria on alumino silicates weathering. American Minerologist. 1998; 83:1551-1563.

6. Deng SB, Bai RB, Hu XM, Luo Q. Characteristics of a bioflocculant produced by Bacillus mucilaginosus and its use in starch waste water treatment. Applied Microbiology and Biotechnology. 2003; 60:588-593.

7. Konga SK. Screening of free living rhizobacteria associated with tobacco rhizosphere for plant growth promoting traits. African Journal of Agricultural Research. 2014; 10(1):194-110.

8. Mikhailouskaya NA, Bogdevitch I. Effect of biofertilizers on yield and quality of long-fibred flax and cereal grains. Agronomy Research. 2009; 7(2):412-418.

9. Patel NM, Sadarai SG, Kaneria BB, Khanpara VD. Effect of nitrogen, potassiuim and zinc on growth and yield of wheat. Indian Journal of Agronomy. 2007; 40(2):90-92.

10. Sheng XF, Xia JJ, Cheng J. Mutagenesis of the Bacillus edaphicus strain NBT and its effect on growth of chilli and cotton. Agriculture Science. 2003; 32(3):258-265.

11. Zhang AM, Zhao GY, Wang W, Li J, Zhang SF, Zhu BC. Solubilization of insoluble potassium and phosphate by Paenibacillus kribensis CX-7: A soil microorganism with biological control potential. African Journal of Microbiology. 2013; 7(1):41-47. 